\title{
Lidil
}

Revue de linguistique et de didactique des langues

38 | 2008

Langue du droit

\section{Apprendre à décrire le document juridique à l'heure du Web 2.0}

\section{Stéphane Cottin}

\section{OpenEdition}

\section{Journals}

Édition électronique

URL : http://journals.openedition.org/lidil/2778

DOI : $10.4000 /$ lidil.2778

ISSN : 1960-6052

\section{Éditeur}

UGA Éditions/Université Grenoble Alpes

\section{Édition imprimée}

Date de publication : 1 décembre 2008

Pagination : 53-70

ISBN : $978-2-84310-130-4$

ISSN : 1146-6480

\section{Référence électronique}

Stéphane Cottin, « Apprendre à décrire le document juridique à l'heure du Web 2.0 », Lidil [En ligne], 38 | 2008, mis en ligne le 01 juin 2010, consulté le 21 avril 2019. URL : http://journals.openedition.org/ lidil/2778; DOI : 10.4000/lidil.2778

Ce document a été généré automatiquement le 21 avril 2019

(C) Lidil 


\title{
Apprendre à décrire le document juridique à l'heure du Web 2.0
}

\author{
Stéphane Cottin
}

Dire que l'accès au droit est de plus en plus complexe est un lieu commun. S'en plaindre, en énumérer les aspects négatifs, proclamer un principe général du droit de sécurité juridique (Documentation française, 2008 ; Article Sécurité Juridique, 2008), un objectif de valeur constitutionnelle d'intelligibilité et d'accessibilité de la Loi (Conseil Constitutionnel, 1999) sont les premières étapes nécessaires pour circonscrire le mal et au moins permettre d'en décrire les symptômes. Trouver des remèdes et convaincre de l'intérêt de les prescrire sont des missions d'urgence, en cours de réalisation, et qu'il convient de suivre avec intérêt, à défaut d'y participer.

1 Mais il semble que l'ensemble de ces réflexions, souvent très avancées, prometteuses et ambitieuses, s'éloignent très vite de ce qui semble être l'essentiel, de l'élément primordial de toutes les constructions juridiques : la définition du document (son identification), sa gestion (notamment sa description), et, si l'on peut se permettre cette mise en abime, le «droit du document juridique ». Il est dommage que les sciences juridiques et les juristes en général se désintéressent autant des sciences documentaires, et plus largement de la bibliothéconomie, voire de la scientométrie, parce que l'inverse n'est pas vrai. Des rapports mutuellement profitables pourraient naitre d'expériences appliquées pratiquement et systématiquement au monde de l'édition juridique, de la conception de la loi ou de l'élaboration de jurisprudence. Des exemples de rencontre existent, ils sont nombreux, mais ne sont ni systématisés, ni souvent poursuivis jusqu'à l'obtention d'un projet viable économiquement, encore moins enseignés ou étudiés dans les facultés de droit.

\section{Faire reculer l'illettrisme informationnel}

Les plus blasés diront qu'il s'agit encore là d'une énième proposition de faire entrer l'enseignement des techniques de la documentation dans les études de droit. Ils rajouteront avec raison que les textes le prévoyaient explicitement ${ }^{1}$, et que certaines 
universités le font. Mais il est généralement reconnu que ces efforts méritoires sont insuffisants au niveau national, et que des générations d'étudiants en droit (et a fortiori leurs enseignants), n'ont pas été correctement formés aux techniques documentaires, et notamment aux nouvelles technologies de la documentation. Aussi est-il normal de ne pas voir émerger de réflexions constructives et permanentes sur le document juridique, les juristes eux-mêmes n'ayant pas été formés aux outils conceptuels modernes de la scientométrie, et ne connaissant que superficiellement (quand ils les connaissent) les méthodes modernes de traitement des documents.

2 On ne peut pas blâmer les étudiants et leurs professeurs de cet illettrisme informationnel: l'apprentissage de ces techniques est complexe et nécessite des infrastructures solides. Le phénomène est connu et de nombreuses initiatives ont déjà été développées, notamment outre-Atlantique : voir le blog collaboratif des documentalistes juridiques canadiens (Slaw.ca, 2008), ou une recherche sur Google par exemple de l'expression « legal information literacy ».

3 La tâche n'est pas aisée, mais personne n'a dit le contraire. Or il devient urgent d'agir, et l'on peut même se demander s'il n'est pas trop tard. Décrire et identifier les documents juridiques sont à la fois des tâches basiques pour tout juriste, mais aussi des missions qui sont l'essence même des techniciens de l'information-documentation, quand, pour eux, cela devient dans leur jargon localiser et acquérir. La différence de vocabulaire pour deux objectifs strictement identiques est symptomatique du fossé qui existe entre les deux métiers.

\section{Difficulté d'accès au document juridique}

Un des rares axiomes les mieux partagés des systèmes juridiques, quelle que soit leur localisation spatio-temporelle, est le caractère préalable de l'édiction de la règle de droit. Ensuite, que cette promulgation soit écrite sur du papier, gravée dans le marbre, ou chantée au son des tambours, le support importe peu : nul n'est censé ignorer la loi. D'aucuns racontent même qu'il s'agit d'un des piliers de l'État de droit, et ils ont sûrement raison.

Or, une autre des vérités premières universellement partagées par l'ensemble des utilisateurs du droit (c'est-à-dire tout le monde), est qu'il est non seulement impossible, mais aussi de plus en plus difficile (nous assumons l'incohérence de la gradation de cette énumération), de tout connaitre en droit.

5 Une question écrite au Sénat (Sénat, 2008), ci-dessous reproduite, exprimait encore une fois cette crainte.

« Nul n'est censé ignorer la loi »; l'adage est bien connu, sauf qu'aujourd'hui, pour que soit appliqué ce principe élémentaire, il faudrait que chacun connaisse les ... 10500 lois, 120000 décrets, 17000 textes communautaires et 7400 traités, que renferment les 62 codes regroupant l'ensemble de l'arsenal législatif et réglementaire ! Considérant que " à l'impossible, nul n'est tenu », M. Georges Mouly demande à $\mathrm{M}$. le Premier ministre, de quelle manière il est possible de simplifier ou de dégrossir cette "inflation législative " qui aboutit, inévitablement, à cet autre adage bien connu : «Trop de loi tue la loi. »

6 Le thème de cette interpellation est régulier : il était en écho à une question de la législature précédente (Sénat, 2006a) à laquelle le Premier ministre avait répondu de façon particulièrement documentée (Sénat, 2007). 
Il entre parmi les devoirs de l'État envers les citoyens de veiller à la sécurité juridique et à la qualité du droit. La recherche de l'efficacité économique (Mission de recherche Droit et Justice, 2005) ${ }^{2}$ ne se conçoit pas sans prise en compte de ces exigences. Le constat des dérives de la production normative que dresse le rapport public $2006 \mathrm{du}$ Conseil d'État ${ }^{3}$ (Conseil d'État, 2006; Sénat, 2006b) rejoint un diagnostic que le Président de la République, les présidents des assemblées et le président du Conseil constitutionnel ${ }^{4}$ (Mazeaud, 2005) ont publiquement exposé au cours des dernières années. Le défi que constitue la lutte contre l'inflation normative et la dégradation de la qualité de la norme appelle, pour une part, une action concertée à l'échelle internationale et plus particulièrement dans le cadre communautaire. Dans le cadre national, le Gouvernement a entrepris de le relever de plusieurs manières. Il rend publics sur le site Internet Légifrance des indicateurs de l'évolution du volume des textes actualisés à un rythme trimestriel (Legifrance, 2006). Un réseau interministériel de hauts fonctionnaires chargés de la qualité de la réglementation est animé depuis 2003 par le secrétariat général du Gouvernement. L'un des objectifs assignés à ce réseau est de veiller à l'application des lois, de même qu'en liaison avec le secrétariat général des affaires européennes, il doit veiller à la transposition des directives. Sur ces deux terrains, des améliorations tangibles sont aujourd'hui perceptibles, contribuant au renforcement de la sécurité juridique. Cette action quotidienne se double d'actions de moyen et de long terme. Le Gouvernement déploie ainsi un vaste programme de simplification du droit. Afin de favoriser l'intégration des préoccupations de qualité du droit dès le stade de la conception de la norme, le secrétariat général du Gouvernement promeut, en concertation avec le Conseil d'État, un guide pour l'élaboration des textes législatifs et réglementaires ${ }^{5}$ (Secrétariat général du Gouvernement, 2007) dont le succès est avéré. Afin de mieux ancrer la pratique des évaluations préalables à l'élaboration des réformes les plus significatives, instruction a été donnée aux ministères d'accompagner tout avant-projet de loi d'une étude d'impact. Par la modernisation $\mathrm{du}$ dialogue social, il entend permettre une meilleure association des partenaires sociaux aux réformes qui les concernent. Enfin, sur la base du rapport public de 2006 du Conseil d'État, le Gouvernement a mené une réflexion circonstanciée sur les modalités des réformes d'ordre institutionnel ou constitutionnel que pourrait justifier l'objectif d'amélioration de la qualité du droit. Un rapport recensant différentes options de réforme et leurs incidences prévisibles a été rendu public ${ }^{6}$ (Secrétariat général du Gouvernement, 2006), notamment sur le site Internet de la Documentation française $^{7}$ et sur Légifrance, de manière à nourrir une réflexion à laquelle ont leur part tant le Gouvernement que le Parlement et la société civile.

7 Le problème est bien envisagé avec le plus grand sérieux par les plus hautes sphères de l'État. Pourtant la situation se complexifie encore plus, car cela fait longtemps que l'on ne raisonne plus ici au niveau local, mais au niveau international.

\section{Systèmes juridiques nationaux et attractivité économique}

En plus de cette contradiction interne déjà particulièrement délétère, se greffe depuis longtemps un constat latent : le droit français ne fait plus « recette ». Bien au-delà, ce sont tous les régimes de droit dits «continentaux", ou «droits (de tradition) romanogermaniques » qui pâtissent de l'influence de plus en plus grande de la Common Law. La concordance des temps permet ici imprudemment de faire le rapprochement avec la fusion, lente mais inexorable, entre les $\mathrm{XIV}^{\mathrm{e}}$ et $\mathrm{XVI}^{\mathrm{e}}$ siècles des systèmes juridiques (Article Systèmes Juridiques, 2008) (Article Droit Coutumier, 2008) des pays de coutume orale en langue d'Oil avec ceux de droit écrit, de droit romain. 
et à l'illustration des systèmes juridiques est «l'attractivité économique " (Mission de recherche Droit et Justice, 2005). "Mondialisation oblige, on insiste davantage aujourd'hui sur les couts de ces désordres normatifs, feignant de découvrir que le droit est un élément de la compétitivité nationale et donc de la prospérité. » (Molfessis, 2006)

plupart de ces réactions, même si elles se situent dans une longue perspective de concurrence comparatiste, ont été récemment relancées par la publication depuis la fin 2003 des rapports Doing Business de la Banque mondiale (Association Henri Capitant, 2008) réplique de l'Association Henri Capitant aux Rapports Doing Business de la Banque Mondiale et (Degos Eversheds, 2008), et plus récemment par un autre phénomène de ranking avec le Classement de Shanghai (classement international des universités, (Article Classement [...] de Shanghai, 2008)) qui placent tous deux la France aux fins fonds de leurs palmarès respectifs.

10 À cet effet, une Fondation pour le droit continental ${ }^{8}$ a été créée pour «renforcer le rayonnement du droit continental et contribuer à l'équilibre juridique mondial ».

\section{Contrôler les instruments de mesure}

En plus des instances et des missions de promotion du droit français à l'étranger, il convient, au moins en interne, d'établir des règles du jeu simples et reconnues par tous les acteurs, comme par exemple en créant un service public. En effet, si la France a décidé depuis près d'un demi-siècle de créer des bases de données officielles pour ses informations juridiques (lois, règlements, jurisprudence, etc.), elle en a conçu dès 1984 un service public de diffusion du droit, désormais, depuis 2002, diffusé par l'Internet. Ce service public, rendu par l'État au profit de tous, consommateurs citoyens, professionnels du droit et éditeurs juridiques, accompagne plusieurs avancées technologiques. La plus importante concerne la dématérialisation du journal officiel électronique authentique (Cottin, 2005).

11 Encore faut-il que les règles soient simples et admises par tout le monde, ce qui n'est pas le cas, même parmi les professionnels de l'information documentation. Le problème le plus emblématique tient dans l'anonymisation des décisions de justice. Celui-ci est largement évoqué dans la notice de contenu de la base de Jurisprudence judiciaire sur le portail Legifrance'. Parmi d'autres nombreux et tout aussi apres débats, nous retiendrons celui sur la diffusion de la jurisprudence autour de l'alternative exhaustivité/sélection, et celui sur la codification autour de la responsabilité éditoriale et de la valeur ajoutée aux données brutes publiées par l'État.

A) Sur la diffusion de la jurisprudence, à l'occasion d'une description savante de l'état de l'art sur une base de données de jurisprudence et de doctrine (Bories, 2003), l'auteur évoquait l'initiative publique de création d'une base de données d'arrêts de Cour d'appel, en proposant de résoudre le débat quantité/qualité dans l'alternative exhaustivité/ sélection en affirmant qu'«il convient de garder à l'esprit que, sans une sélection de l'information fondée sur des critères qualitatifs, nous assisterions à la création de fichiers pléthoriques, entrainant inévitablement un nivellement corrélatif de l'information et un lourd déficit de sens $»^{10}$.

12 Proposer ce principe de sélection entre en contradiction directe avec les pratiques généralement constatées sur l'Internet où l'exhaustivité semble devenir le principe, et 
avec les besoins d'une partie non négligeable des utilisateurs finaux, qui désireraient justement une accessibilité pleine et entière aux données brutes. Cette sélection est évidemment utile aux universitaires ou au grand public, car elle facilite la compréhension ou l'apprentissage du droit. Toutefois les avocats, les magistrats, tous les professionnels $\mathrm{du}$ droit ressentent aussi le besoin de se reposer sur l'ensemble des sources du droit, d'être sûrs d'avoir accès à l'intégralité des données proposées et non à des résumés, des sélections ou des extraits. Ces utilisateurs ont peut-être tort, cela ne cadre certainement pas avec l'exigence de l'article 5 du Code civil réprimant les " arrêts de règlement " $^{11}$, cela n'est pas politiquement correct dans le cadre international de soutien du droit écrit contre la Common Law, mais c'est une réalité professionnelle vécue, et que tous les documentalistes juridiques connaissent.

B) Sur la codification, nous constatons que les critiques sont étonnamment nombreuses autour de l'effort public de codification. Elles sont aussi parfois contradictoires. Il est reproché d'une part de ne pas aller assez loin, ni assez vite, dans l'effort de reconstruction, de quasi-refondation du droit que supposent les entreprises de codification. Les différentes circulaires, dont la plus complète est celle du 30 mai 1996 relative à la codification des textes législatifs et réglementaires (Juppé, 1996), prévoyaient par exemple un programme achevé en 2000, objectif qui n'a pu être atteint.

D'autre part, une certaine catégorie de représentants de la doctrine reproche aussi à la codification d'aboutir à une impasse, et même d'être néfaste (Moysan, 2004). Les éditeurs juridiques privés considèrent aussi que ce travail de codification, souvent confondu avec celui de "consolidation " (travail de réécriture en temps réel des textes en vigueur en fonction des modifications apportées par le législateur ou le pouvoir exécutif), leur fait concurrence: selon eux, il ne devrait appartenir à l'État que de fournir des données brutes, et c'est au secteur privé concurrentiel de réaliser les produits à valeur ajoutée.

Sur les très vives critiques émises par la doctrine à l'encontre de l'entreprise de codification menée depuis 1989, nous constatons le ton pour le moins polémique, sinon pessimiste, des titres des articles ci-dessous, à défaut de leur contenu, parmi une littérature abondante :

- A. Bied-Charreton, « Un procédé qui verse dans l'abstraction » (entretien) : La Tribune, 21 nov. 2003, p. 25 ;

- D. Bureau et N. Molfessis, «Le nouveau Code de commerce : une mystification »: D. 2001, chron. $n^{\circ} 17$, p. 361 ;

- S. Guy, « La codification : une utopie » : RFD const. 1996, p. 273 ;

- N. Molfessis, «Les illusions de la codification à droit constant et la sécurité juridique » : RTD civ. 2000, p. 186 ;

- «Le Code civil et le pullulement des codes » dans Le Code civil : un passé, un présent, un avenir, Dalloz, 2004 ;

- H. Moysan, «La codification à droit constant ne résiste pas à l'épreuve de la consolidation » : Dr. adm. 2002, chron. $n^{\circ} 7$ et JCP 2002, G, I, nº 147 ;

- G. Taormina, « La codification est-elle encore utile ? Éléments pour une méthodologie historique »: RRJ 2002, $n^{\circ} 1$, p. 22 ;

- F. Terré et A. Outin-Adam, « Codifier est un art difficile (à propos d'un... code de commerce)» : D. 1994, chron., p. 99.

Ces deux vifs débats sont loin d'être les seuls qui opposent, parfois à front renversé, les différents acteurs de la documentation juridique, qu'ils soient producteurs ou consommateurs, car ils sont souvent les deux à la fois. 


\section{Profiter avec efficacité des nouvelles technologies}

Il apparait bien que la plupart des acteurs français sont en train de passer à côté de nombreux et utiles apports des nouvelles technologies de l'information documentation, faute d'en appréhender les avantages économiques et/ou de peur d'y perdre leur âme, ou pire, de l'argent.

16 Il est envisageable par exemple d'utiliser les outils du web 2.0 en créant des réseaux sociaux, et en y étudiant les interactions de diverses jurisprudences (Gallacher, 2007).

17 Il est possible d'assister à des cours de droit virtuels à distance, via Second Life (Hudson, 2008) ou de podcaster du droit (ServiceDoc.Info, 2007a), notamment les cours du Collège de France $^{12}$, les émissions hebdomadaires d'Antoine Garapon ${ }^{13}$ ou les chroniques matinales d'Olivier Duhamel ${ }^{14}$, pour ne citer que quelques trop rares exemples qui mériteraient d'être multipliés.

18 Un Conseil national du droit ${ }^{15}$ a été, en avril 2008, « chargé d'une mission de réflexion et de proposition sur l'enseignement du droit, sur les relations entre les établissements qui dispensent cet enseignement et les institutions et professions concernées, sur la formation et l'emploi des juristes et sur les orientations et les modalités de la recherche juridique ».

19 Le domaine du traitement documentaire des données juridiques, et surtout celui de la formation de tous les utilisateurs (producteurs et consommateurs) potentiels de ces données, semble entrer dans les attributions de ce conseil. Comme aucun professionnel de l'information-documentation, aucun bibliothécaire, aucun éditeur juridique, n'est convié à participer aux réflexions du conseil suscité, il serait alors judicieux d'offrir à cet aréopage de professeurs quelques pistes de réflexion.

\section{Projet de feuille de route pour améliorer l'identification et la gestion des documents juridiques}

Ces améliorations peuvent se situer tant en amont qu'en aval de la chaine de production documentaire. De façon cursive et quasi-chronologique, on pourra relever ainsi les suggestions suivantes :

1) Améliorer l'écriture du droit :

- pour les normes, la légistique.

20 La légistique est la "(science appliquée) de la législation, qui cherche à déterminer les meilleures modalités d'élaboration, de rédaction, d'édiction et d'application des normes ». (Chevallier, 1995).

Déjà évoqué plus haut à propos de la codification, le secrétariat général du Gouvernement $(\mathrm{SGG})$ a mis en ligne sa circulaire d'aide à l'élaboration des textes normatifs. Cela n'est pas tout à fait une informatisation de la méthode, mais une intrusion utile des techniques informatiques dans les travaux dévolus aux collaborateurs du SGG pour réaliser des textes réglementaires ou aider à élaborer des textes législatifs.

Répartis en fiches didactiques, régulièrement et scrupuleusement mises à jour, les documents sont aussi publiés à la Documentation française (Secrétariat général du 
Gouvernement, 2007). Ils sont intégralement en ligne dès la page d'accueil du portail Legifrance ${ }^{16}$.

- pour les jurisprudences, la jurimétrique.

L'actualité du domaine de l'informatique appliquée à la jurisprudence est très riche, le lecteur se reportera par exemple à Paré (2008). Pour avoir une idée du grand nombre d'articles publiés sur le thème depuis 1963, le lecteur se reportera au I. de la bibliographie de la thèse de Jean Leclerc (1999).

- pour la doctrine, la scientométrie ou bibliométrie.

24 «La bibliométrie se définit comme l'exploitation statistique des publications. Cette analyse permet de rendre compte de l'activité des producteurs (chercheur, laboratoire, institut...) ou des diffuseurs (périodique, éditeur...) de l'information scientifique, tant d'un point de vue quantitatif que qualitatif. $\aleph^{17}$ Pour une application aux sciences juridiques, voir Cottin (1997).

2) Améliorer la formation des acteurs :

- formation continue aux nouvelles technologies des professionnels du droit déjà en activité ;

- formation initiale des étudiants aux outils innovants;

- incitation des chercheurs à se tourner vers ces nouveaux horizons de recherche, tant en linguistique qu'en organisation, ou en mathématiques appliquées aux sciences sociales.

3) Faciliter l'accès des acteurs aux outils simples du web 2.0 en assurant notamment la promotion des réseaux sociaux :

- usage des listes de signets partagés, comme avec l'outil delicio.us ${ }^{18}$;

- partage de fils de syndication de contenu (ou « rss ») sur des univers netvibes ${ }^{19}$;

- développement des réseaux spécialisés collaboratifs sous forme de nuages de blogs, de réseaux sociaux de type «ning $»^{20}$, d'encyclopédies collaboratives comme la Jurispedia ${ }^{21}$.

4) Mettre au point un ensemble de principes simples et normalisés de description de document juridique :

- des URI (Uniform Resource Identifier) ou DOI (Digital Object Identifier) stables (Bermès, 2006) ;

- référence neutre pour les normes et les jurisprudences. Pour l'exemple du comité canadien de la référence neutre, voir (ServiceDoc.Info, 2003);

- normalisation généralisée des indications bibliographiques au niveau des éditeurs et des universitaires ${ }^{22}$;

- stabilisation des systèmes de licences de rediffusion de l'ensemble des documents juridiques ${ }^{23}$.

5) Constituer un catalogue des ressources disponibles : identifier, cartographier et valider ces ressources :

- identifier : nécessite au préalable la normalisation des outils de description ;

- cartographier : archivage ouvert de l'ensemble des corpus à identifier ${ }^{24}$;

- valider : assurer un suivi, une cohérence et une fiabilité absolue de ces sources, mais aussi s'assurer de la valeur et des droits moraux et patrimoniaux sur chacune de ces sources.

La constitution de ce catalogue pourrait être du ressort de l'Agence du patrimoine immatériel de l'État (APIE) ${ }^{25}$. Il est déclaré en effet sur la page d'accueil du site de l'agence qu' ' au titre de ses premières actions, [elle] pilotera un recensement d'ensemble des actifs immatériels des administrations et des établissements publics de l'État ».

26 À noter que l'article 17 de la loi du 17 juillet $1978^{26}$ tel que modifié par l'article 10 de l'ordonnance $\mathrm{n}^{\circ}$ 2005-650 du 6 juin 2005 dispose désormais que ${ }^{27}$ : 
«Les administrations qui produisent ou détiennent des informations publiques tiennent à la disposition des usagers un répertoire des principaux documents dans lesquels ces informations figurent.

Les conditions de réutilisation des informations publiques, ainsi que les bases de calcul retenues pour la fixation du montant des redevances, sont communiquées, par les administrations qui ont produit ou détiennent ces informations, à toute personne qui en fait la demande.»

Cet article est précisé par l'article 36 du décret n 2005-1755 du 30 décembre 2005 relatif à la liberté d'accès aux documents administratifs et à la réutilisation des informations publiques, pris pour l'application de la loi $\mathrm{n}^{\circ} 78-753$ du 17 juillet $1978^{28}$ :

«Le répertoire prévu à l'article 17 de la loi du 17 juillet 1978 susvisée précise, pour chacun des documents recensés, son titre exact, son objet, la date de sa création, les conditions de sa réutilisation et, le cas échéant, la date et l'objet de ses mises à jour. Lorsque l'autorité administrative dispose d'un site internet, elle rend le répertoire accessible en ligne. »

6) Permettre d'enrichir ces ressources:

- élaboration d'un ou de thésaurus et d'ontologies permettant de décrire ces corpus ;

- réindexation rétrospective des données : nécessite un plan préalable (mis en commun) de récupération au moins des références des ressources, sinon de la numérisation rétrospective de ces ressources.

\section{Conclusion}

La quête de l'objectif d'intelligibilité et d'accessibilité du droit nécessite des efforts de tous les acteurs, qu'ils soient producteurs ou consommateurs de documents juridiques.

Depuis l'élaboration de chaque document juridique (que ce soit une norme, une jurisprudence ou de la doctrine), à sa réception par les professionnels du droit ou son application par les citoyens/administrés, en passant par ses différents traitements: publication, archivage, modification, etc., il est primordial d'assurer une identification parfaite, simple et non équivoque à cet objet. Pour ce faire, les pouvoirs publics producteurs, les intermédiaires traitants, les utilisateurs finaux, professionnels, étudiants ou simples citoyens, doivent disposer d'offres documentaires et de méthodes fiables et reconnues. Si plusieurs de ces offres sont déjà disponibles (Legifrance, le guide de légistique...), les méthodes sont en cours d'élaboration et pourraient arriver bientôt à maturité, pour peu que la feuille de route proposée soit au moins partiellement suivie.

\section{BIBLIOGRAPHIE}

ASSOCIATION HENRI CAPITANT (2008) : Les droits de tradition civiliste en question (vol. 1 et 2). À propos des Rapports « Doing Business » de la Banque Mondiale, Association Henri Capitant <http:// www.fondation-droitcontinental.org/6.aspx?sr=705>.

Article Sécurité Juridique (2008) : <http://fr.wikipedia.org/wiki/Sécurité_juridique>, Wikipedia. 
Article Systèmes Juridiques (2008) : <http://fr.wikipedia.org/wiki/Système_juridique>, Wikipedia.

Article Droit Coutumier (2008) : <http://fr.wikipedia.org/wiki/Droit_coutumier>, Wikipedia.

Article Classement académique des universités mondiales par l'université Jiao Tong de Shanghai (2008) : <http://fr.wikipedia.org/wiki/

Classement_académique_des_universités_mondiales_par_l'université_Jiao_Tong_de_Shanghai>, Wikipedia.

BERMÈs E. (2006) : Emmanuelle Bermès, Bibliothèque nationale de France, Des identifiants

pérennes pour les ressources numériques. L'expérience de la BnF : < $\underline{\text { http://bibnum.bnf.fr/ }}$ identifiants/identifiants-200605.pdf>.

BORIES S. (2003) : L'informatisation des données judiciaires et doctrinales : une contribution à la connaissance et à la recherche juridiques, Documentaliste - Sciences de l'information, oct. 2003, vol. $40, \mathrm{n}^{\circ} 4-5$, p. 272-279.

BORIES S. (2002) : Quelles revues pour le $21^{\mathrm{e}}$ siècle, Revue trimestrielle de droit civil, oct.-déc. 2002, $\mathrm{n}$ - 4, p. 714.

CHEVALLIER J. (1995) : L'évaluation législative : un enjeu politique, dans Alain Delcamp et al., Contrôle parlementaire et évaluation, Paris, 1995, p. 15.

CONSEIL CONSTITUTIONNEL (1999) : Cons. const. n 99-421 DC du 16 décembre 1999, Loi portant habilitation du Gouvernement à procéder, par ordonnances, à l'adoption de la partie législative de certains codes ; <http://www.conseil-constitutionnel.fr/decision/1999/99421/99421dc.htm>. CONSEIL D'ÉTAT (2006) : Rapport public 2006 du Conseil d'État, Sécurité juridique et complexité du droit, Études et Documents du Conseil d'État, 57, Paris : La documentation française, 416 p., <http:// www.conseil-etat.fr/ce/rappor/index_ra_li0600.shtml>.

COTTIN S. (1997) : La bibliométrie appliquée aux articles de doctrine juridique Un exemple autour des articles de doctrine sur les décisions du Conseil constitutionnel. Revue de la Recherche Juridique - Droit Prospectif (1997/3), 837-853. <http://www.servicedoc.info/La-bibliometrie-appliquee-aux,165>. CoTTIN S. (2005) : Le service public de diffusion du droit français sur l'Internet, Acta Universitatis Sibiu (Roumanie), $\mathrm{n}^{\circ}$ 1-2/2005, <http://www.servicedoc.info/Le-service-public-de-diffusion$\mathrm{du}, 1703>$.

DEGOS EVERSHEDS L. (2008) : La Lettre des juristes d'affaires, 11/02/2008 concurrence des places de droit, <http://www.fondation-droitcontinental.org/6.aspx?sr=704>.

DOCUMENTATION FRANÇAISE (2008) : Dossier Qualité des normes et sécurité juridique, site Internet de la Documentation française : <http://www.ladocumentationfrancaise.fr/dossiers/qualite-normessecurite-juridique/securite-juridique.shtml>.

GALLACHER I. (2007) : Syracuse University College of Law. « Mapping the Social Life of the Law: An Alternative Approach to Legal Research », The Selected Works of Ian Gallacher <http:// works.bepress.com/ian_gallacher/10>.

GRIMALDI M. (2002) : Quelles revues pour le $21^{\mathrm{e}}$ siècle ?, Revue trimestrielle de droit civil, oct.-déc. $2002, \mathrm{n}^{\circ} 4$, p. 714 .

HUDSON R. (2008) : A Little Grafting of Second Life into a Legal Research Class, Rob Hudson (Head of Information Services, Nova Southeastern University, Shepard Broad Law Center - Law Library \& Technology Center, Fort Lauderdale, FL.), Legal Librarian Research eXchange, 9 mai 2008. 
JUPPÉ A. (1996) : Journal officiel du 5 Juin 1996, p. 8263, <http://www.legifrance.gouv.fr/jopdf/ common/jo_pdf.jsp?numJO=0\&dateJO=19960101\&pageDebut $=08263>$.

LECLERC J. (1999) : Les représentations informatiques des connaissances juridiques, Thèse Lille 2, 1999, publiée sur le Village de la Justice : <http://www.village-justice.com/articles/RepresentationsInformatiques, $526 . \mathrm{html}$.

LEGIFRANCE.GOUV.FR (2006) : Rubrique « Évolution du volume des textes » du site Legifrance, <http:// www.legifrance.gouv.fr/html/statistiques_normatives/liste_stats_essai.htm>.

MAZEAUD P. (2005) : Échange de vœux à l'Élysée le 3 janvier 2005, <http://www.conseilconstitutionnel.fr/bilan/annexes/voeuxpr2005.htm>.

MISSION DE RECHERCHE DROIT ET JUSTICE (2005), Programme international de recherche « Attractivité économique du droit ", <http://www.gip-recherche-justice.fr/aed.htm>.

MOLFESSIS N. (2006) : Combattre l'insécurité juridique ou la lutte du système juridique contre lui-même, Rapport annuel 2006 du Conseil d'État, p.390 ; Conseil d'État, Rapport public 2006 « Sécurité juridique et complexité du droit ».

MOYSAN H. (2004), La consolidation des codes, lois et décrets : positions doctrinales d'éditeurs ou devoir de l'État? (objectif de valeur constitutionnelle d'intelligibilité et d'accessibilité de la loi), dans Colloque Law via the Internet 2004: $6^{e}$ journées internationales Internet pour le Droit 2004, Paris, < http://www.frlii.org/article.php3?id_article=68>.

PARÉ E. (2008) : La demi-vie des jugements, Lex Electronica, vol. 13, n 1, Printemps / Spring 2008, < http://www.lex-electronica.org/articles/v13-1/pare.htm>.

SECRÉTARIAT GÉNÉRAL du GOUVERNEMENT (2006) : Rapport au Premier Ministre du groupe de travail chargé d'une réflexion sur les suites du rapport public 2006, <http://www.conseil-etat.fr/ce/ rappor/pdf/suite_rapport_ce_2006.pdf>.

SECRÉTARIAT GÉNÉRAL DU GOUVERNEMENT, CONSEIL D'ÉTAT (2007) : Guide de Légistique, guide pour l'élaboration des textes législatifs et réglementaires, $2^{\mathrm{e}}$ éd. 2007, <http://www.legifrance.gouv.fr/ html/Guide_legistique/accueil_guide_leg.htm>.

sÉNAT (2006a) : Question écrite $n^{\circ} 24774$ de M. Jean-Marc Pastor(Tarn - SOC) publiée dans le JO Sénat du 12 octobre 2006, p. 2577, <http://www.senat.fr/basile/visio.do?id=qSEQ061024774>.

sÉNAT (2006b) : Question écrite n 22614 de M. Marcel Vidal (Hérault - SOC) publiée dans le JO Sénat du 6 avril 2006, p. 960 et réponse du Premier ministre publiée dans le JO Sénat du 22 juin 2006, p.1709, <http://www.senat.fr/basile/visio.do?id=qSEQ060422614>.

sÉNAT(2007) : Réponse du Premier ministre publiée dans le JO Sénat du 15 février 2007, p. 332, < http://www.senat.fr/basile/visio.do?id=qSEQ061024774>.

sÉNAT (2008) : Question écrite $n^{\circ} 04335$ de M. Georges Mouly (Corrèze - RDSE), publiée dans le JO Sénat du 8 mai 2008, p. 897, <http://www.senat.fr/basile/visio.do?id=qSEQ080504335>.

SERVICEDOC.INFO (2003) : Le comité canadien de la référence, 17 août 2003, <http:// www.servicedoc.info/La-reference-neutre-le-comite,106>.

SERVICEDOC.INFO (2007a) : Du droit à podcaster, 28 août 2007, < http://www.servicedoc.info/Du-droita-podcaster,2039.html>.

SERVICEDOC.INFO (2007b) : Complexité du droit et sécurité juridique : les moyens d'action du gouvernement, 16 février 2007, <http://www.servicedoc.info/Complexite-du-droit-et-securite,1999>. 
SLAW.CA (2008): Kim Nayyer, Associate and National Research Lawyer, Miller Thomson LLP, Toronto (9 mars 2008): The Information Literate Legal Researcher, <http://www.slaw.ca/2008/03/09/ the-information-literate-legal-researcher/>.

\section{NOTES}

1. L'article 8 de l'arrêté du 30 avril 1997 relatif au diplôme d'études universitaires générales Droit et aux licences et aux maîtrises du secteur Droit et science politique (sic) (Journal officiel, $\mathrm{n}$ -104 du 4 mai 1997, p.6766, <http://www.legifrance.gouv.fr/affichTexte.do? cidTexte=JORFTEXT000000382767>) disposait que « La première année du DEUG Droit débute par un semestre d'orientation [comprenant] trois unités d'enseignement :

[...] - une unité de méthodologie du travail universitaire dont la durée représente 15 à $20 \%$ du volume horaire du semestre. Cette unité contribue à promouvoir l'autonomie des étudiants et leur fournit les méthodes et techniques utiles à la poursuite d'études (préparer une bibliographie, utiliser une bibliothèque et les nouvelles sources d'information, prendre des notes, résumer un article ou un ouvrage... ».

2. 〈http://www.gip-recherche-justice.fr/aed.htm>.

3. <http://www.conseil-etat.fr/ce/rappor/index_ra_li0600.shtml>.

4. <http://www.conseil-constitutionnel.fr/bilan/annexes/voeuxpr2005.htm>.

5. <http://www.legifrance.gouv.fr/html/Guide_legistique/accueil_guide_leg.htm>.

6. $<$ http://www.conseil-etat.fr/ce/rappor/pdf/suite_rapport_ce_2006.pdf>.

7. <http://www.ladocumentationfrancaise.fr/rapports-publics/074000100/index.shtml> ; à noter aussi, cité sur la page du site du Conseil d'État consacrée à son rapport 2006, précité, <http:// www.conseil-etat.fr/ce/rappor/index_ra_li0600.shtml>, l'étude: Inventaire méthodique et codification du droit de la communication <http://www.conseil-etat.fr/ce/rappor/ index_ra_li0601.shtml>, réalisée pour le compte de la Commission supérieure de la codification, dans lequel on préconise notamment l'ouverture d'un portail numérique susceptible d'améliorer rapidement, hors de toute codification formelle, l'accessibilité et l'intelligibilité du droit: < http://www.ladocumentationfrancaise.fr/catalogue/9782110061652/>.

8. <http://www.fondation-droitcontinental.org/>.

9. <http://www.legifrance.gouv.fr/html/aide/aide_contenu_juris_judiciaire.htm>.

10. Serge Bories (2002) cite aussi : «Michel Grimaldi, dans une formule qui peut être généralisée à tous les professionnels du droit, écrit, à propos de la profession notariale : “Aussi n'a-t-il guère besoin d'une information massive, mais d'une information significative" (Grimaldi, 2002). »

11. Article 5 du Code civil : "Il est défendu aux juges de prononcer par voie de disposition générale et réglementaire sur les causes qui leur sont soumises. »

12. 〈http://www.college-de-france.fr/default/EN/all/pub_pod/index.htm>.

13. <http://www.radiofrance.fr/chaines/france-culture2/emissions/bien_commun/>.

14. <http://radiofrance-podcast.net/podcast/rss_18811.xml>.

15. <Décret $\mathrm{n}^{\circ} 2008-420$ du 29 avril 2008 portant création du Conseil national du droit, <http:// www.legifrance.gouv.fr/affichTexte.do?dateTexte=\&cidTexte=JORFTEXT000018740489>.

16. <http://www.legifrance.gouv.fr/html/Guide_legistique/accueil_guide_leg.htm>.

17. Définition issue du dossier "Bibliométrie» de la Médiathèque scientifique de l'Institut Pasteur, <http://www.pasteur.fr/infosci/biblio/services/metrie/>.

18. <http://delicious.com/tag/recherche_doc $>$ ou <http://delicious.com/tag/droit>.

19. Tout comme il existe déjà l'univers netvibes de la Documentation française, < http:// www.netvibes.com/ladoc> : communiqué de presse sur < $\underline{\text { ttp:// }}$ 
www.ladocumentationfrancaise.fr/informations/presse/2008/df-netvibes.pdf>, on verra aussi un exemple d'univers de documentaliste juridique sur $<$ http://www.netvibes.com/juriconnexion>.

20. <http://juriblog.ning.com>.

21. <http://fr.jurispedia.org>.

22. Un groupe de travail du SNE (Syndicat national de l'édition) travaille sur les questions liées au traitement de l'information juridique et notamment l'harmonisation des abréviations des principales références en matière juridique. < http://www.sne.fr/pdf/ Abr_princ_references_juridiques.pdf>.

23. <http://www.legifrance.gouv.fr/html/licences/menu_licences.htm>. Voir aussi les Rapports annuels du comité du service public de la diffusion du droit par l'Internet sur <http:// www.legifrance.org/html/apropossite/aproposdusite.htm>.

24. À rapprocher des initiatives de numérisation rétrospective, comme lors de cette journée d'information organisée par la BNF sur la numérisation en sciences juridiques <http:// www.bnf.fr/PAGES/infopro/journeespro/jp_num_juridique.htm>, ou cet appel à contributions lancé sur le blog du Professeur Rolin le 14 décembre 2007, <http://frederic-rolin.blogspirit.com/ archive/2007/12/04/la-bibliographie-des-ouvrages-a-numeriser-dans-le-cadre-du-p.html>.

25. 〈http://www.minefe.gouv.fr/directions_services/apie/index.htm>.

26. Loi $n^{\circ} 78-753$ du 17 juillet 1978 modifiée portant diverses mesures d'amélioration des relations entre l'administration et le public et diverses dispositions d'ordre administratif, social et fiscal, <http://www.legifrance.gouv.fr/affichTexte.do?cidTexte=LEGITEXT000006068643>. $<\quad$ http://www.legifrance.gouv.fr/affichTexteArticle.do? idArticle=LEGIARTI000006528251\&cidTexte=LEGITEXT000006068643>.

28. JORF, $\mathrm{n}^{\circ} 304$ du 31 décembre 2005, p. 20827, texte $\mathrm{n}^{\circ}$ 119, <http://www.legifrance.gouv.fr/ affichTexte.do?cidTexte=JORFTEXT000000265304\&dateTexte=\#JORFSCTA000000898939>.

\section{RÉSUMÉS}

L'inflation documentaire dans le domaine du droit est un phénomène indéniable auquel sont confrontés tous les acteurs juridiques au sens large. Malgré les initiatives du gouvernement français pour faciliter l'accès à certaines sources juridiques (Légifrance en est un exemple), l'accès à l'information et au document juridiques est de plus en plus complexe, d'autant plus complexe qu'il existe une forme d'illettrisme informationnel parmi les professionnels du droit. En contrepoint de cette complexité exponentielle, force est de constater que les entreprises font jouer la concurrence des systèmes juridiques du point de vue de leur attractivité économique. Devant la nécessité d'améliorer l'identification et la gestion des documents juridiques, nous proposons une feuille de route pour améliorer la boite à outil primordiale pour toute construction et/ou de tout raisonnement juridique.

Documentary inflation in the field of Law is an undeniable phenomenon with which all legal actors are confronted. In spite of the initiatives of the French government to facilitate access to certain legal sources (Legifrance is an example), access to legal information and documents is increasingly complex, all the more complex as there exists a form of informational illiteracy among legal professionals. Above and beyond this exponential complexity, multinational companies/businesses/corporations put into play competition between legal systems in terms of their respective economic attractiveness. Faced with the need to improve the identification and 
management of legal documents, we suggest a roadmap to improve the necessary toolbox for any legal construction and/or reasoning.

\section{AUTEUR}

\section{STÉPHANE COTTIN}

Secrétariat Général du Gouvernement 